\title{
Black phosphorus-based van der Waals heterostructures for mid-infrared light-emission applications
}

Xinrong Zong ${ }^{1,2}$, Huamin $\mathrm{Hu}^{3}$, Gang Ouyang ${ }^{3}$, Jingwei Wang ${ }^{4}$, Run Shi', Le Zhang ${ }^{1}$, Qingsheng Zeng ${ }^{5}$, Chao Zhu ${ }^{5}$, Shoukeng Chen ${ }^{1}$, Chun Cheng $\mathbb{1}^{4}$, Bing Wang ${ }^{6}$, Han Zhang $\mathbb{1}^{6}$, Zheng Liu $\mathbb{1}^{5}$, Wei Huang ${ }^{2,7}$, Taihong Wang ${ }^{1}$, Lin Wang ${ }^{2}$ and Xiaolong Chen ${ }^{1}$

\begin{abstract}
Mid-infrared (MIR) light-emitting devices play a key role in optical communications, thermal imaging, and material analysis applications. Two-dimensional (2D) materials offer a promising direction for next-generation MIR devices owing to their exotic optical properties, as well as the ultimate thickness limit. More importantly, van der Waals heterostructures_combining the best of various 2D materials at an artificial atomic level-provide many new possibilities for constructing MIR light-emitting devices of large tuneability and high integration. Here, we introduce a simple but novel van der Waals heterostructure for MIR light-emission applications built from thin-film BP and transition metal dichalcogenides (TMDCs), in which BP acts as an MIR light-emission layer. For BP-WS $e_{2}$ heterostructures, an enhancement of $\sim 200 \%$ in the photoluminescence intensities in the MIR region is observed, demonstrating highly efficient energy transfer in this heterostructure with type-I band alignment. For BP-MoS 2 heterostructures, a room temperature MIR light-emitting diode (LED) is enabled through the formation of a vertical PN heterojunction at the interface. Our work reveals that the BP-TMDC heterostructure with efficient light emission in the MIR range, either optically or electrically activated, provides a promising platform for infrared light property studies and applications.
\end{abstract}

\section{Introduction}

As an emerging member of the two-dimensional (2D)layered material family, black phosphorus $(\mathrm{BP})^{1-6}$ has been widely studied for its unique properties, such as inplane anisotropy ${ }^{5,7}$, infrared bandgap energy ${ }^{8-10}$, and high carrier mobility ${ }^{11-14}$, which enable wide applications in electronic and optoelectronic devices ${ }^{15,16}$. Due to the

Correspondence: Taihong Wang (wangth@sustech.edu.cn) or

Lin Wang (iamlwang@njtech.edu.cn) or Xiaolong Chen (chenxl@sustech.edu.cn)

${ }^{1}$ Department of Electrical and Electronic Engineering, Southern University of

Science and Technology, 518055 Shenzhen, China

${ }^{2}$ Key Laboratory of Flexible Electronics (KLOFE) \& Institute of Advanced Materials

(IAM), Jiangsu National Synergetic Innovation Center for Advanced Materials

(SICAM), Nanjing Tech University (Nanjing Tech), 30 South Puzhu Road, 211816

Nanjing, China

Full list of author information is available at the end of the article

These authors contributed equally: Xinrong Zong, Huamin Hu, Gang Ouyang efficiently tuneable bandgap energy through thickness $(0.3-2 \mathrm{eV} \text { ) and electric-field (down to } 0.05 \mathrm{eV})^{17-21}$ modulation, thin-film BP is considered a promising midinfrared (MIR) material, filling the energy gap between semimetallic graphene and semiconducting transition metal dichalcogenides (TMDCs) $(1.0-2.5 \mathrm{eV})^{22}$. Utilizing the MIR properties of thin-film BP, optoelectronic devices, such as MIR photodetectors and optical modulators with high performance, have been demonstrated ${ }^{17,22-27}$.

A decent light-emission property is also crucial for photonic and optoelectronic device applications. Previous reports have focused on the visible and near-infrared photoluminescence (PL) properties of monolayer and few-layer BP $(<5 \text { layers })^{9,28-31}$. Until very recently, the MIR PL of thin-film BP was investigated by Chen et al., revealing that thin-film BP is a promising material for

\section{(c) The Author(s) 2020}

(c) (i) Open Access This article is licensed under a Creative Commons Attribution 4.0 International License, which permits use, sharing, adaptation, distribution and reproduction cc) in any medium or format, as long as you give appropriate credit to the original author(s) and the source, provide a link to the Creative Commons license, and indicate if changes were made. The images or other third party material in this article are included in the article's Creative Commons license, unless indicated otherwise in a credit line to the material. If material is not included in the article's Creative Commons license and your intended use is not permitted by statutory regulation or exceeds the permitted use, you will need to obtain permission directly from the copyright holder. To view a copy of this license, visit http://creativecommons.org/licenses/by/4.0/. 
MIR light-emission applications ${ }^{8}$. To achieve higher efficiency and lower power-consumption devices, thin-film $\mathrm{BP}$ with a better light-emission density is necessary. In addition, more emission driving modes are also desirable. For example, electrically driven MIR light emission (electroluminescence/EL) is more favorable for practical photonic and optoelectronic applications.

Due to the high-quality interface and lack of lattice mismatch, vdWs heterostructures built from 2D-layered materials, such as graphene and TMDCs, have been intensively investigated for various applications, including transistors $^{32}$, solar cells ${ }^{33}$, photodetectors ${ }^{34}$, and lightemitting devices ${ }^{35}$. In recent years, BP-based vdWs heterostructures have begun to attract great attention due to their narrow bandgap and anisotropic lattice structure. For example, $\mathrm{BP}-\mathrm{MoS}_{2}$ heterostructures have enabled high-performance photodetectors ${ }^{36}$ and high-gain logic inverters ${ }^{37-39}$. BP-graphene heterostructures can sustain a large pseudomagnetic field at the interface ${ }^{40}$.

Here, we introduce a high-quality van der Waals (vdWs) heterostructure targeted for MIR light-emission applications constructed from thin-film BP and TMDCs, such as monolayer tungsten diselenide ( $\left.\mathrm{WSe}_{2}\right)$ and thin-film molybdenum disulfide $\left(\mathrm{MoS}_{2}\right)$. Combining density functional theory (DFT) calculations ${ }^{41}$ and experimental observations, a type-I band alignment is formed in the $\mathrm{BP}-\mathrm{WSe}_{2}$ heterostructure, and efficient energy transfer from $\mathrm{WSe}_{2}$ to thin-film BP is enabled. As a result, a $192 \%$ enhancement of the MIR PL is observed at a wavelength of $2.79 \mu \mathrm{m}$, and this enhancement effect persists up to $3.89 \mu \mathrm{m}$. On the other hand, a PN heterojunction tuneable by a source-drain voltage is achieved in the $\mathrm{BP}-\mathrm{MoS}_{2}$ heterostructure, with which an MIR light-emitting diode (LED) is demonstrated. In addition, highly anisotropic PL and EL are observed in these $\mathrm{BP}-\mathrm{WSe}_{2}$ and $\mathrm{BP}-\mathrm{MoS}_{2}$ heterostructures, respectively. All the results suggest that constructing BP-TMDC heterostructures is an efficient and facile strategy for MIR light-emission investigations and applications.

\section{Results}

\section{Mid-infrared photoluminescence enhancement in $\mathrm{BP}-\mathrm{WS} \mathrm{e}_{2}$ heterostructures}

Figure 1 shows the DFT-calculated electron affinity and bandgap size of monolayer $\mathrm{WSe}_{2}$ and BP. The detailed band structures are further illustrated in Supplementary Fig. 1. The bandgap size of BP decreases with increasing thickness, consistent with previous theoretical calculations and experimental observations ${ }^{6,15,41}$. In addition, BP shows a direct bandgap at all thicknesses, suggesting that it is a promising material for light-emission applications. To realize its application in the MIR region $(2.5-25 \mu \mathrm{m})$, we select thin-film BP with a layer number larger than six to construct the heterostructure. According to the DFT

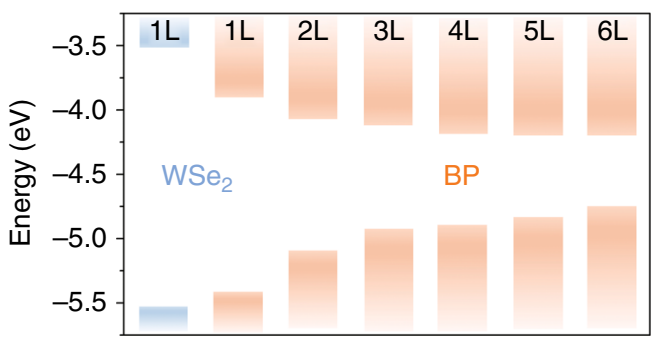

Fig. 1 Density functional theory investigation of $\mathrm{BP}-\mathrm{WSe}_{2}$ heterostructures. Band diagram of monolayer $\mathrm{WS}_{2}$ and $\mathrm{BP}$ (with layer numbers from one to six) calculated by the HSE06 functional. Type-l band alignment is formed in the BP-WSe $e_{2}$ heterostructures

calculation, the $\mathrm{BP}-\mathrm{WSe}_{2}$ heterostructure forms a type-I band alignment. In this band alignment, thin-film BP serves as a quantum well and can efficiently collect electron and hole pairs from adjacent monolayer $\mathrm{WSe}_{2}{ }^{42}$. On the other hand, monolayer $\mathrm{WSe}_{2}$ has a high optical absorption and excellent quantum efficiency in the visible region (complementary to the MIR range of thin-film $\mathrm{BP})^{43}$. Hence, it is an ideal optical absorption layer for enhancing the luminescence efficiency of BP in terms of the absorption wavelength, recombination efficiency, and energy transfer.

Figure 2a shows a schematic diagram of the BP-TMDC heterostructure. The bottom TMDC layer has a hexagonal crystalline structure, with a layer of tungsten/molybdenum atoms sandwiched between two selenium/sulfur atom layers ${ }^{22}$. The phosphorus atoms in each layer of BP form a folded anisotropic honeycomb structure ${ }^{15}$. The $x$ and $y$ directions are used to denote the armchair and zigzag crystalline directions of $\mathrm{BP}$, respectively. To build this heterostructure, first, TMDC flakes and thin-film BP were mechanically exfoliated onto $285-\mathrm{nm} \mathrm{SiO}_{2} / \mathrm{Si}$ and polydimethylsiloxane (PDMS) substrates, respectively. Then, the thin-film BP was transferred onto the TMDCs using the PDMS-assisted transfer method ${ }^{44}$. To enhance the vdWs interactions between BP and the TMDCs, the heterostructures were further heated at a temperature of $200^{\circ} \mathrm{C}$ for $10 \mathrm{~min}$. All operations were performed in a glovebox filled with nitrogen to avoid BP surface oxidation and achieve a high-quality vdWs interface. In this work, the BP flakes were aligned with the TMDC layers with random angles, and all the samples showed PL enhancement in the MIR range. However, the alignment angle between BP and the TMDCs can possibly influence the detailed emission properties of the heterostructure, which is outside the scope of this article. Further studies on this effect are highly encouraged and will benefit the heterostructure 2D materials community. Figure $2 \mathrm{~b}$ shows an optical image of a typical $\mathrm{BP}-\mathrm{WSe}_{2}$ heterostructure sample. The contours of the monolayer $\mathrm{WSe}_{2}$ and thin-film BP flakes are outlined by orange and blue 

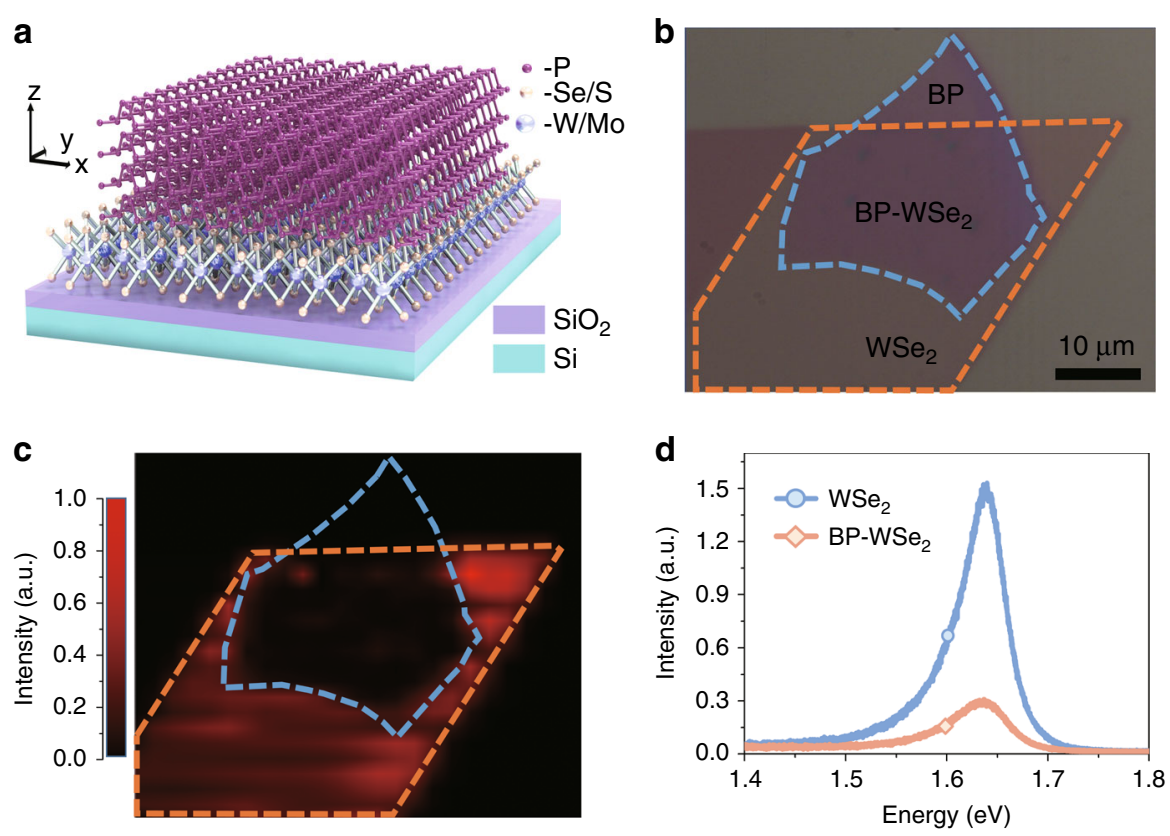

Fig. 2 Configuration and visible photoluminescence of $\mathrm{BP}-\mathrm{WSe}_{\mathbf{2}}$ heterostructures. a-c Schematic (a), optical (b), and photoluminescence (PL) mapping (c) images of the BP-WSe 2 heterostructure. The scale bar is $10 \mu \mathrm{m}$. d PL spectra of monolayer WSe $\mathrm{S}_{2}$ and the $\mathrm{BP}-\mathrm{WS}_{2}$ heterostructure under an incident laser of $2.33 \mathrm{eV}$. The incident laser power is $4 \mu \mathrm{W}$

dashed lines, respectively. The thickness of thin-film BP is $\sim 6 \mathrm{~nm}$, as determined by atomic force microscopy (AFM) and PL measurements (see Supplementary Fig. 2). The Raman spectra of the $\mathrm{BP}-\mathrm{WSe}_{2}$ stack show the characteristic vibration modes of both thin-film BP and monolayer $\mathrm{WSe}_{2}$ (see Supplementary Fig. 3), indicating successful preparation of the heterostructure.

We first examined the PL properties of the $\mathrm{BP}-\mathrm{WSe}_{2}$ heterostructure in the visible region under $2.33-\mathrm{eV}$ laser excitation. Figure $2 \mathrm{c}$ shows the PL emission mapping of the sample at room temperature. High PL intensity is observed in the monolayer $\mathrm{WSe}_{2}$ region due to its high light absorption and quantum efficiency, consistent with previous studies ${ }^{45,46}$. On the other hand, significant PL quenching is observed in the $\mathrm{BP}-\mathrm{WSe}_{2}$ heterojunction area. As shown in Fig. 2d, the PL intensity in the heterostructure region decreases by $80 \%$ compared with that of monolayer $\mathrm{WSe}_{2}$. This observation agrees with our theoretical prediction of the type-I band alignment between monolayer $\mathrm{WSe}_{2}$ and $\mathrm{BP}$, which enables efficient carrier transfer from the wide bandgap $\mathrm{WSe}_{2}$ to the narrow bandgap BP. As a result, the photogenerated electron and hole densities in $\mathrm{WSe}_{2}$ decrease, leading to a significant PL intensity reduction in the heterostructure region. Another argument is that the PL intensity reduction for $\mathrm{WSe}_{2}$ in the heterostructure region is due to the light absorption of the top BP layer. To exclude this possibility, we assembled another $\mathrm{BP}-\mathrm{WSe}_{2}$ heterostructure under ambient conditions with a 7.5-nm-thick
BP flake. Due to the reaction of BP with water and oxygen during the fabrication process under ambient conditions, a thin phosphorus oxide $\left(\mathrm{P}_{\mathrm{x}} \mathrm{O}_{\mathrm{y}} \sim 2 \mathrm{~nm}\right)$ will form at the $\mathrm{BP}-\mathrm{WSe}_{2}$ interface, resulting in a $\mathrm{BP}-\mathrm{P}_{\mathrm{x}} \mathrm{O}_{\mathrm{y}}-\mathrm{WSe}_{2}$ threelayer structure ${ }^{20,47}$. The interface oxide layer can effectively prevent charge transfer between $\mathrm{BP}$ and $\mathrm{WSe}_{2}$, and hence, a small reduction of the visible PL intensity should be observed in the heterostructure region. In this device, only a $33 \%$ reduction of the PL intensity is observed in the heterostructure region (see Supplementary Fig. 4), indicating that the contribution from the light absorption by the top BP layer is insignificant.

To further demonstrate the efficient energy transfer in the $\mathrm{BP}-\mathrm{WSe}_{2}$ heterostructure, we characterized its MIR light-emission properties under 2.33-eV laser excitation. The incident laser power was fixed at $20 \mu \mathrm{W} \mu \mathrm{m}^{2}$ with a laser spot diameter of $\sim 15 \mu \mathrm{m}$. PL studies at additional incident laser powers are shown in Supplementary Fig. 5. Figure 3a shows the MIR PL spectra of the BP film and $\mathrm{BP}-\mathrm{WSe}_{2}$ heterostructure region, where the $\mathrm{BP}$ thickness is approximately $5 \mathrm{~nm}$. We achieve a $165 \%$ enhancement of the MIR PL intensity in the heterostructure region, which is defined as $\left(I_{\mathrm{BP}-\mathrm{WSe} 2}-I_{\mathrm{BP}}\right) / I_{\mathrm{BP}}$. Here, $I_{\mathrm{BP}-\mathrm{WSe} 2}$ and $I_{\mathrm{BP}}$ are the MIR PL intensities of the $\mathrm{BP}$ and $\mathrm{BP}-\mathrm{WSe}_{2}$ heterostructure regions, respectively. In addition, the PL peaks of the two regions are at the same position of $\sim 3.18 \mu \mathrm{m}$, indicating that carriers transferred from monolayer $\mathrm{WSe}_{2}$ are effectively confined in the BP quantum well and then recombined with MIR light 

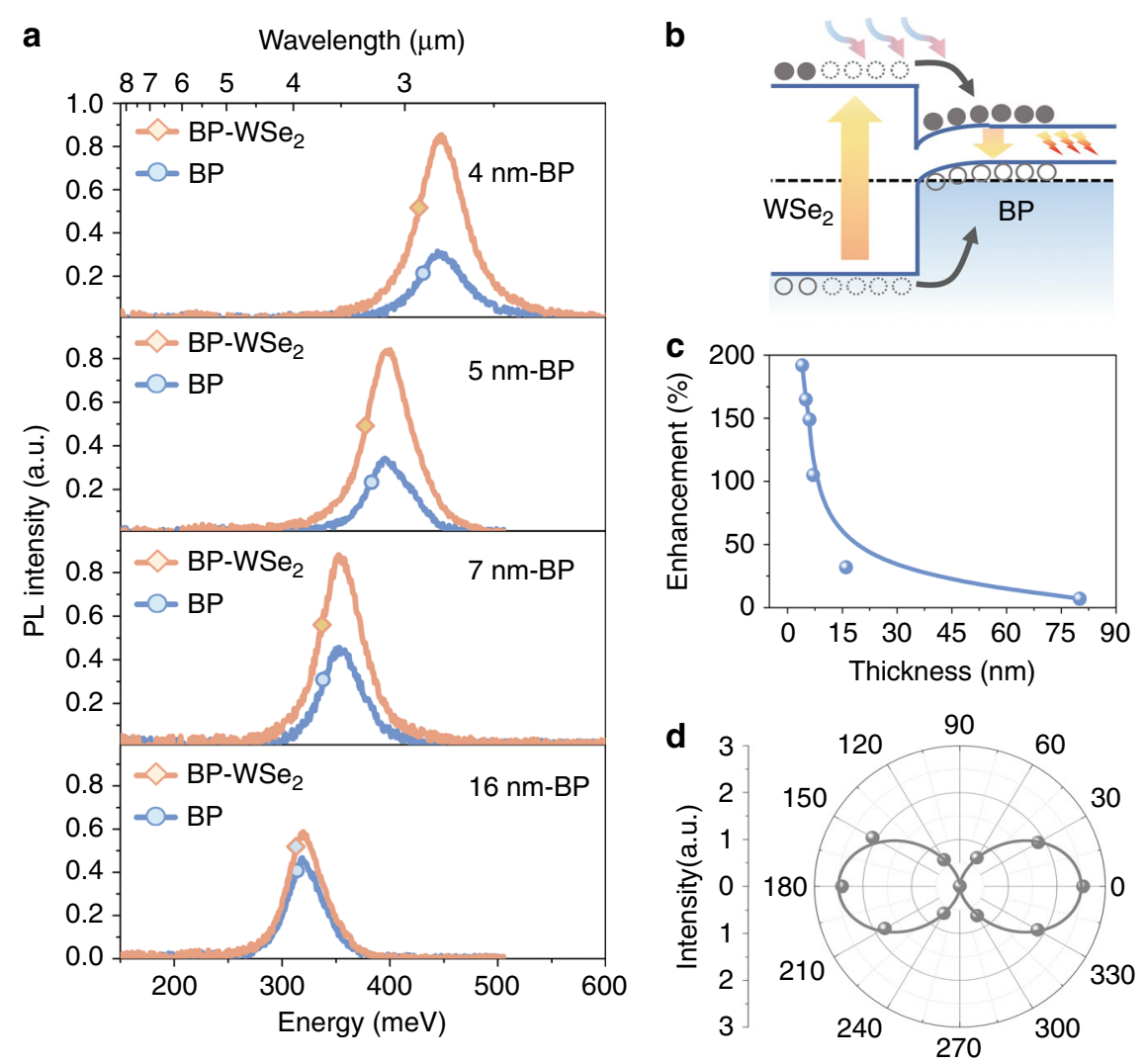

Fig. 3 MIR photoluminescence spectra of BP-WSe ${ }_{2}$ heterostructures. a MIR PL spectra of thin-film BP (blue lines) and BP-WSe $e_{2}$ heterostructures (orange lines) at $80 \mathrm{~K}$. $\mathbf{b}$ Schematic band diagram of $\mathrm{BP}-\mathrm{WSe}_{2}$ heterostructures. The dashed line denotes the Fermi energy of $\mathrm{BP}$ and WSe $2 . \mathbf{c} \mathrm{MIR} \mathrm{PL}$ enhancement in $\mathrm{BP}-\mathrm{WSe}_{2}$ heterostructures as a function of thickness. The solid line is a guide line. $\mathbf{d}$ Polarization-resolved MIR PL spectra for the $\mathrm{BP}-\mathrm{WS}_{2}$ heterostructure with 5 -nm-thick BP. The solid line is the fitting curve obtained using the equation $I=\left(I_{\max }-I_{\min }\right) \cos ^{2} \theta+I_{\min }$. Here, $\theta$ is the polarization angle referenced to the armchair axis of $\mathrm{BP}$, and $I_{\max }$ and $I_{\min }$ are the $\mathrm{PL}$ intensities along the armchair-and zigzag axes, respectively

emission, as illustrated in Fig. 3b. For the $\mathrm{BP}-\mathrm{P}_{\mathrm{x}} \mathrm{O}_{\mathrm{y}}-\mathrm{WSe}_{2}$ heterostructure sample fabricated in air, the enhancement at the heterostructure is only $\sim 25 \%$. This is due to the presence of the phosphorus oxide at the interface, which significantly reduces the carrier transfer rate from monolayer $\mathrm{WSe}_{2}$ to BP. In addition, we characterized the enhancement of the MIR PL intensity at additional laserexcitation energies. Enhancement factors of $138 \%$ and $116 \%$ are achieved at excitation energies of $1.95 \mathrm{eV}$ and $1.81 \mathrm{eV}$, respectively, as shown in Supplementary Fig. 6 . We attribute the excitation energy-dependent enhancement factor to the optical absorption of monolayer $\mathrm{WSe}_{2}$. A higher absorption can generate more electron-hole pairs and thus result in a larger enhancement factor. At a laser-excitation energy of $1.58 \mathrm{eV}$, which is below the exciton energy of monolayer $\mathrm{WSe}_{2}$, no enhancement effect is observed in the heterostructure region. This phenomenon provides direct evidence that type-I band alignment is achieved in the $\mathrm{BP}-\mathrm{WSe}_{2}$ heterostructure.

We further performed MIR PL measurements of $\mathrm{BP}-\mathrm{WSe}_{2}$ heterostructures with $\mathrm{BP}$ thicknesses ranging from $4 \mathrm{~nm}$ to $80 \mathrm{~nm}$ at $80 \mathrm{~K}$. As shown in Fig. 3a, c, we achieve a PL enhancement over a broad MIR region from $2.79 \mu \mathrm{m}$ to $3.89 \mu \mathrm{m}$. For example, a $192 \%$ enhancement is obtained in the heterostructure with 4-nm-thick BP (see Fig. 3c). For the 16-nm-thick BP, the enhancement is $\sim 32 \%$. The PL enhancement of the heterostructures gradually decreases as the thickness of BP increases. This phenomenon can be attributed to two reasons. On the one hand, the MIR emission from the $\mathrm{BP}-\mathrm{WSe}_{2}$ heterostructure is contributed by two components $\left(I=I_{\mathrm{s}}+I_{\mathrm{t}}\right)$. The component $I_{\mathrm{s}}$ comes from the self-generated electron-hole pairs in $\mathrm{BP}$, and $I_{\mathrm{t}}$ comes from the electron-hole pairs transferred from monolayer $\mathrm{WSe}_{2}$. It is obvious that higher $I_{\mathrm{t}}$ and smaller $I_{\mathrm{s}}$ will lead to a larger enhancement effect. For thicker BP flakes, the ratio between $I_{\mathrm{t}}$ and $I_{\mathrm{s}}$ decreases because thicker BP absorbs more light and the bottom $\mathrm{WSe}_{2}$ layer absorbs less light. On the other hand, the band bending of $\mathrm{BP}$ near the $\mathrm{BP}-\mathrm{WSe}_{2}$ interface can separate the electrons and holes, as shown in Fig. 3b. Thicker BP flakes will lead to a larger spatial separation between electrons and holes and will 
hence decrease the electron-hole recombination efficiency. As a result, the enhancement factor is reduced for thicker BP flakes. We also plot the MIR PL peak position of the $\mathrm{BP}-\mathrm{WSe}_{2}$ heterostructures as a function of $\mathrm{BP}$ thickness and temperature, and the results are in good agreement with previous reports on thin-film $\mathrm{BP}^{8}$ (see Supplementary Figs. 7 and 8).

The highly anisotropic MIR light emission is still preserved in the $\mathrm{BP}-\mathrm{WSe}_{2}$ heterostructures, as demonstrated by the polarization-resolved PL spectra shown in Fig. 3d. Here, the laser-excitation direction is fixed along the armchair axis of BP, and the detection angle $\theta$ is the intersection angle between the detection direction and the armchair axis of BP.

\section{Mid-infrared electroluminescence in $\mathrm{BP}-\mathrm{MoS}_{2}$ heterojunction diodes}

In contrast to the $\mathrm{BP}-\mathrm{WSe}_{2}$ heterostructure, the $\mathrm{BP}-\mathrm{MoS}_{2}$ heterostructure forms a type-II band alignment according to previous experimental observations ${ }^{36,37}$. In addition, thin-film BP and $\mathrm{MoS}_{2}$ always show p-type and n-type semiconducting characteristics, respectively, due to the presence of defects ${ }^{1,2,39,48}$. As a result, a PN heterojunction is naturally formed at the $\mathrm{BP}-\mathrm{MoS}_{2}$ interface, and a diode can be built from this heterostructure. Through tuning of the source-drain voltage, the transfer of electrons from the $\mathrm{MoS}_{2}$ conduction band to the BP conduction band is possible, enabling electrically driven MIR light emission in BP.

Figure $4 \mathrm{a}, \mathrm{b}$ shows a schematic and optical images of a $\mathrm{BP}-\mathrm{MoS}_{2}$ heterojunction diode, respectively. The thickness of the BP flake is $\sim 60 \mathrm{~nm}$, as determined by AFM (see Supplementary Fig. 2). We chose 7-nm-thick $\mathrm{MoS}_{2}$ instead of a monolayer, taking advantage of the higher carrier mobility in thin-film $\mathrm{MoS}_{2}{ }^{32}$. We first characterized the transport properties of thin-film BP and $\mathrm{MoS}_{2}$. As demonstrated by the transfer curves in Fig. 4c, thin-film $\mathrm{BP}$ and $\mathrm{MoS}_{2}$ exhibit p-type and n-type characteristics, respectively. The weak gate tuneability of the BP conductance can be attributed to the larger thickness of $\sim 60 \mathrm{~nm}$, which will screen the gate-field effect. The linear drain-source current-voltage curves $\left(I_{\mathrm{ds}}-V_{\mathrm{ds}}\right)$ indicate that ohmic contacts are achieved between the metal electrodes $(\mathrm{Cr} / \mathrm{Au} 5 / 60 \mathrm{~nm}$ ) and 2D flakes (see Supplementary Fig. 9).

The $I_{\mathrm{ds}}-V_{\mathrm{ds}}$ characterization of the $\mathrm{BP}-\mathrm{MoS}_{2}$ heterojunction diode at room temperature is shown in Fig. $4 \mathrm{~d}$. Under a negative $V_{\mathrm{ds}}$, band-to-band tunneling of charge carriers from the $\mathrm{MoS}_{2}$ conduction band to the BP valence band is possible (see Fig. 5a). Hence, a large current is observed under negative $V_{\mathrm{ds}}$. This band-toband tunneling phenomenon has also been observed in previous studies of $\mathrm{BP}-\mathrm{ReS}_{2}, \mathrm{BP}-\mathrm{SnSe}_{2}$, and $\mathrm{BP}-\mathrm{MoS}_{2}$ heterostructures $^{39,47,49}$. Under a positive $V_{\mathrm{ds}}$, the conduction band of $\mathrm{MoS}_{2}$ bends downward, and electrons accumulate near the $\mathrm{MoS}_{2}$ surface, while the valence band of BP bends upward, and holes accumulate near the BP surface (see Fig. 5b). Since the barrier height is lowered for $V_{\mathrm{ds}}>0$, thermionic electrons from the conduction band of $\mathrm{MoS}_{2}$ to that of BP are enabled. On the other hand, holes in BP are confined near the BP surface due to the larger barrier height $(\sim 1 \mathrm{eV})$. As a result, active electron and hole recombination will occur in thin-film BP. In addition, the drain-source current $I_{\mathrm{ds}}$ can be further tuned by the gate voltage $V_{\mathrm{g}}$. For a positive $V_{\mathrm{g}}$, the electron concentrations in $\mathrm{MoS}_{2}$ are higher, leading to a larger $I_{\mathrm{ds}}$, while the Fermi energy of BP is less affected by $V_{\mathrm{g}}$ due to the electron screening in $\mathrm{MoS}_{2}$.

The EL spectra of the $\mathrm{BP}-\mathrm{MoS}_{2}$ heterojunction diode at $80 \mathrm{~K}$ and $300 \mathrm{~K}$ are shown in Fig. 5c. At $80 \mathrm{~K}$, the EL spectrum is maximized at a wavelength of $4.09 \mu \mathrm{m}$. Importantly, the EL still persists at room temperature. The abnormal blueshift of the EL spectra at higher temperatures can be attributed to the temperature-induced strain effect in BP, consistent with previous observations of temperature-dependent PL spectra of thin-film $\mathrm{BP}^{8}$. The intensity of the EL spectra shows a good linear relation with the injected current, as shown in Fig. 5d. Similar to the PL spectra of BP, the EL spectra also show highly anisotropic characteristics. The EL intensity reaches the maximum (minimum) value when the detection direction is along the armchair axis (zigzag-axis) of BP. The EL intensity ratio between the armchair axis and zigzag-axis is over 7 (see Fig. 5e).

\section{Discussion}

In summary, we have shown that $\mathrm{BP}-\mathrm{TMDC}$ heterostructures $\left(\mathrm{BP}-\mathrm{WSe}_{2}\right.$ and $\left.\mathrm{BP}-\mathrm{MoS}_{2}\right)$ are promising candidates for MIR light-emission applications. For the $\mathrm{BP}-\mathrm{WSe}_{2}$ heterostructures, a type-I band alignment is formed, and a large enhancement of the PL intensities in the MIR region is observed due to the efficient energy transfer from $\mathrm{WSe}_{2}$ to BP. In addition, the PL of the $\mathrm{BP}-\mathrm{WSe}_{2}$ heterostructures also shows strong polarization and thickness dependences. For the BP- $\mathrm{MoS}_{2}$ heterostructures, the type-II band alignment enables the formation of a PN heterojunction at the interface. Based on this heterostructure, an MIR LED has been further realized at room temperature, which fills a gap in the research field of 2D-material-based LEDs.

\section{Materials and methods}

\section{Theoretical method}

Density functional theory (DFT) calculations were performed using the generalized gradient approximation (GGA) of Perdew-Burke-Ernzerhof (PBE) as implemented in the Vienna Ab initio Simulation Package (VASP). The hybrid Heyd-Scuseria-Ernzerhof (HSE06) 

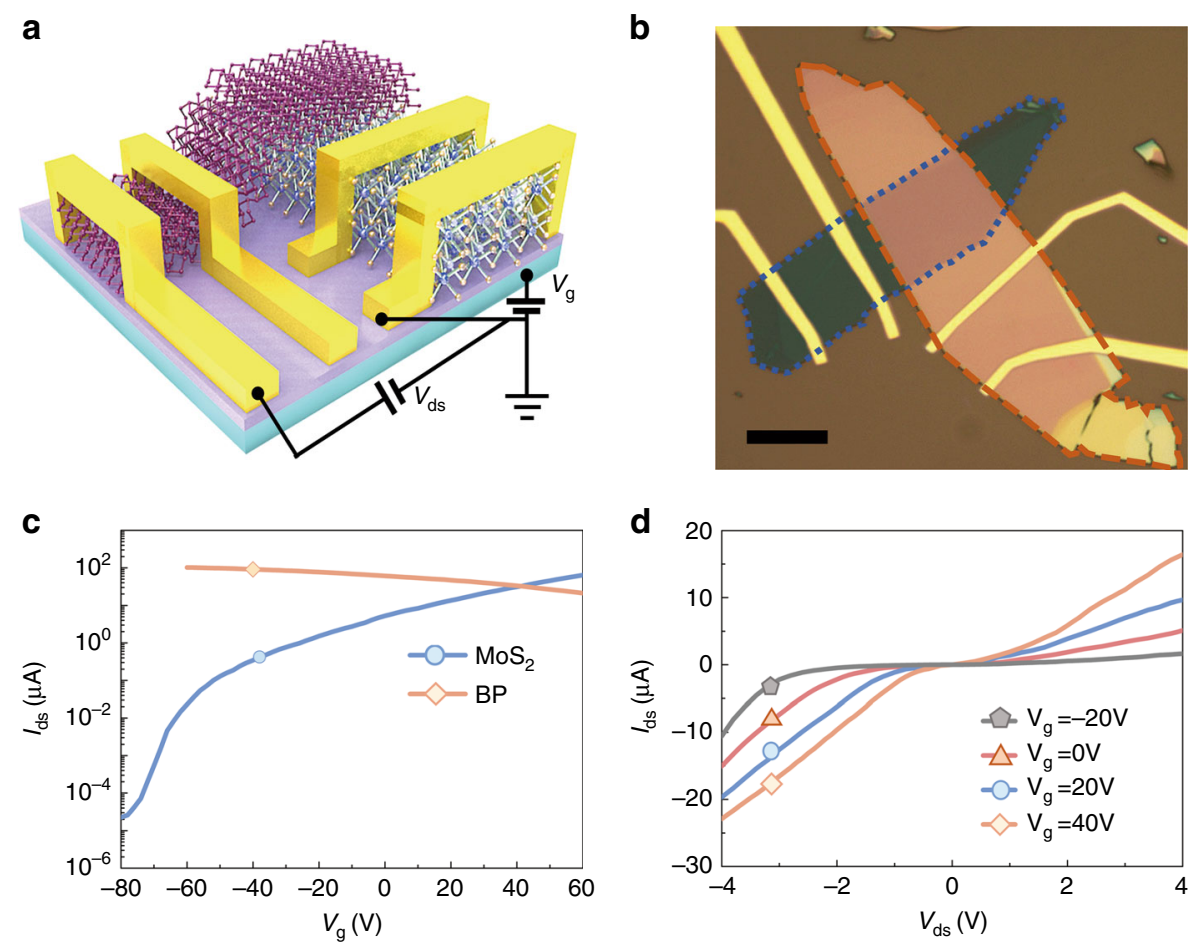

Fig. 4 Configuration and electrical characterization of the BP-MoS heterojunction diode. $\mathbf{a}, \mathbf{b}$ Schematic (a) and optical (b) images of the $\mathrm{BP}-\mathrm{MOS}_{2}$ heterojunction diode. The scale bar is $10 \mu \mathrm{m} . \mathrm{MoS}_{2}$ and BP flakes are enclosed by blue and orange dashed lines, respectively. c Transfer curves of thin-film $\mathrm{MoS}_{2}$ (blue line) and BP (orange line) at source-drain voltage $V_{\mathrm{ds}}=0.5 \mathrm{~V}$ at room temperature. $\mathbf{d}$ Source-drain current $I_{\mathrm{ds}}$ as a function of $V_{d s}$ at various gate voltages $V_{g}$ for the $\mathrm{BP}-\mathrm{MoS}_{2}$ heterojunction diode at room temperature
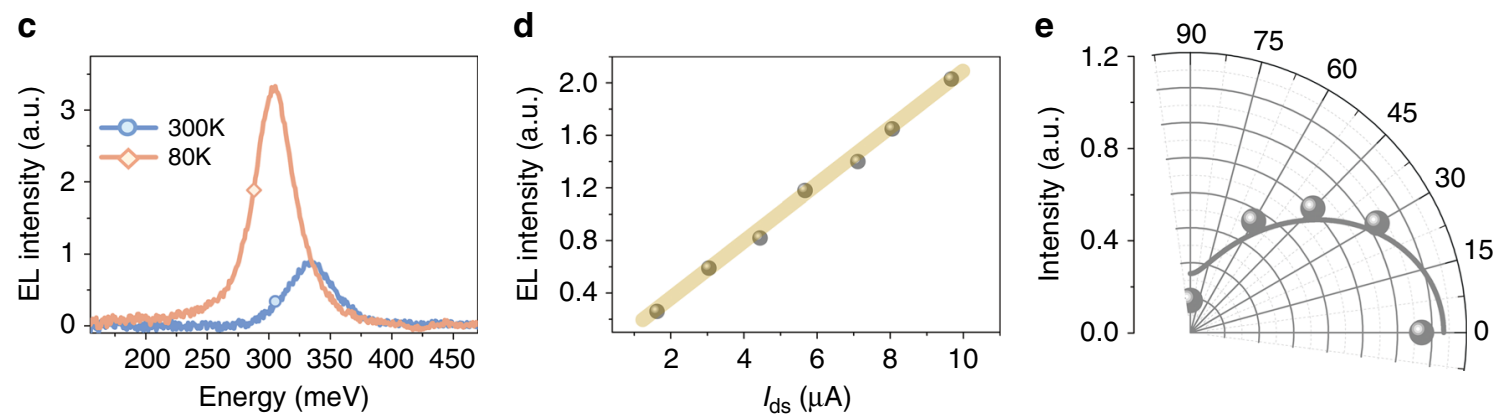

Fig. 5 Mid-infrared electroluminescence in the BP-MoS $\mathbf{2}$ heterojunction diode. $\mathbf{a}$, $\mathbf{b}$ Schematic band diagram of the BP-MoS 2 heterojunction diode for $V_{\mathrm{ds}}<0, V_{\mathrm{g}}=0$ (a) and $V_{\mathrm{ds}}>0, V_{\mathrm{g}}>0$ (b). c EL at $80 \mathrm{~K}, I_{\mathrm{ds}}=8.05 \mu \mathrm{A}$ (orange line) and at $300 \mathrm{~K}, I_{\mathrm{ds}}=8.50 \mu \mathrm{A}$ (blue line). $\mathbf{d} \mathrm{EL}$ intensity as a function of source-drain current $I_{\mathrm{ds}}$ when $V_{\mathrm{ds}}>0$. The yellow solid line serves as a guide line. e Polarization-resolved EL emission at $80 \mathrm{~K}$ and $I_{\mathrm{ds}}=$ $8.05 \mu \mathrm{A}$. The solid line is the fitting curve obtained using the equation $I=\left(I_{\max }-I_{\min }\right) \cos ^{2} \theta+I_{\min }$. Here, $\theta$ is the polarization angle referenced to the armchair axis of BP, and $I_{\max }$ and $I_{\min }$ are the EL intensities along the armchair- and zigzag-directions, respectively 
functional was selected to calculate the band structure and band alignments of BP-TMDC heterostructures. The optB88-vdW functional correction was used to describe the long-range vdWs interaction. A cutoff energy of $400 \mathrm{eV}$ was set for the plane wave expansion. The convergence criterion of energy was set to $10^{-6} \mathrm{eV}$, and that of the force on each atom was less than $0.01 \mathrm{eV}^{-1}$. The vacuum layer height along the $z$ direction was set to $15 \AA$ to avoid interactions between two adjacent images. Monkhorst-Pack k-point grids of $12 \times 12 \times 1$ and $10 \times$ $12 \times 1$ were used in the first Brillouin zone for $\mathrm{WSe}_{2}$ and $\mathrm{BP}$, respectively.

\section{Sample preparation}

TMDC flakes were prepared on a $285-\mathrm{nm}-\mathrm{SiO}_{2} / \mathrm{Si}$ substrate with the standard mechanical exfoliation method in an atmospheric environment. Meanwhile, thin-film BP was mechanically exfoliated from bulk BP crystals onto a PDMS/glass substrate. BP was then overlaid onto the $\mathrm{WSe}_{2}$ flake by the PDMS-assisted dry transfer technique under an optical microscope in a glovebox ${ }^{44}$. The heterostructure samples were further heated at a temperature of $200^{\circ} \mathrm{C}$ for $10 \mathrm{~min}$ in the glovebox to increase the vdWs interactions between BP and the TMDC flakes. To prevent oxidation and photodegradation $^{50}$, all processes involving $\mathrm{BP}$ were performed in a nitrogen-filled glovebox.

\section{Optical characterizations}

For visible PL measurements, the samples were placed in a vacuum chamber. PL and Raman spectroscopies were performed in a confocal HORIBA LabRAM system equipped with 600 grooves per millimeter gratings. Related measurements were carried out at room temperature using a $\times 50$ objective, and the incident laser was $532 \mathrm{~nm}$ with a power fixed at $4 \mu \mathrm{W}$. Due to the glass layer between the objective lens and the sample, the diameter of the laser spot focused on the sample was $2-4 \mu \mathrm{m}$. For MIR PL and EL spectroscopies, samples were placed on a low-temperature stage coupled with a Bruker FTIR spectrometer and a Hyperion 2000 microscope. The MIR PL and EL signals were collected using the lock-in scheme as reported in previous studies ${ }^{9}$, which can significantly reduce the random thermal noise from the environment. For MIR PL measurement, a 533-nm incident laser was chopped at a frequency of $10 \mathrm{kHz}$, and the laser spot size on the sample was $\sim 15 \mu \mathrm{m}$. The incident power was fixed at $20 \mu \mathrm{W} \mu \mathrm{m}^{-2}$. A Stanford Research SR830 was used to lock the frequency and coupled to the FTIR spectrometer. For MIR EL measurement, a sinusoidal voltage with a frequency of $1 \mathrm{kHz}$ and a peak-to-peak voltage of $30 \mathrm{~V}$ was applied to the gate of the $\mathrm{BP}-\mathrm{MoS}_{2}$ heterojunction diode. As a result, the EL spectra were modulated to an AC signal, and random thermal noise could be filtered by the lock-in amplifier. To reduce the effect of $\mathrm{CO}_{2}$ absorptions at an MIR wavelength of $4.3 \mu \mathrm{m}$, the system was purged with $\mathrm{N}_{2}$ gas for $1 \mathrm{~h}$ before PL and EL measurements (see Supplementary Fig. 10).

\section{Acknowledgements}

The work was financially supported by the National Natural Science Foundation of China (Grant Nos. 61904077, 61801210, 91833302, 11574080, 61904080), the Natural Science Foundation of Jiangsu Province (Grant Nos. BK20180686, BK20190670, BK20161008), the funding for "Distinguished professors" and "High-level talents in six industries" of Jiangsu Province (Grant No. XYDXX-021), the Fundamental Research Funds for the Central Universities, and the start-up foundation of Nanjing Tech University and Northwestern Polytechnical University.

\section{Author details}

${ }^{1}$ Department of Electrical and Electronic Engineering, Southern University of Science and Technology, 518055 Shenzhen, China. ${ }^{2}$ Key Laboratory of Flexible Electronics (KLOFE) \& Institute of Advanced Materials (IAM), Jiangsu National Synergetic Innovation Center for Advanced Materials (SICAM), Nanjing Tech University (Nanjing Tech), 30 South Puzhu Road, 211816 Nanjing, China. ${ }^{3}$ Key Laboratory of Low-Dimensional Quantum Structures and Quantum Control of Ministry of Education, and Key Laboratory for Matter Microstructure and Function of Hunan Province, Hunan Normal University, 410081 Changsha, China. ${ }^{4}$ Department of Materials Science and Engineering, Southern University of Science and Technology, 518055 Shenzhen, China. ${ }^{5}$ Center for

Programmable Materials School of Materials Science and Engineering Nanyang Technological University, Singapore 639798, Singapore. ${ }^{6}$ Institute of Microscale Optoelectronics, Collaborative Innovation Centre for Optoelectronic Science \& Technology, Key Laboratory of Optoelectronic Devices and Systems of Ministry of Education and Guangdong Province, College of Physics and Optoelectronic Engineering, Shenzhen Key Laboratory of Micro-Nano Photonic Information Technology, Guangdong Laboratory of Artificial Intelligence and Digital Economy (SZ), Shenzhen University, 518060 Shenzhen, China. ${ }^{7}$ Frontiers Science Center for Flexible Electronics (FSCFE), Shaanxi Institute of Flexible Electronics (SIFE) \& Shaanxi Institute of Biomedical Materials and Engineering (SIBME), Northwestern Polytechnical University (NPU), 127 West Youyi Road, 710072 Xi'an, China

\section{Author contributions}

X.C. and L.W. conceived and supervised the projects. X.Z. fabricated devices. X.Z., J.W., and R.S. performed device characterizations. H.H. and G.O. performed the theoretical modeling. Q.Z., C.Z., and Z.L. prepared the single crystals of BP, $\mathrm{WSe}_{2}$, and $\mathrm{MoS}_{2}$. X.C., L.W., and X.Z. drafted the paper. All authors discussed and commented on the paper.

\section{Data availability}

The data that support the findings of this study are available from the corresponding author upon reasonable request.

\section{Conflict of interest}

The authors declare that they have no conflict of interest.

Supplementary information is available for this paper at https://doi.org/ 10.1038/s41377-020-00356-x.

Received: 10 February 2020 Revised: 14 June 2020 Accepted: 18 June 2020 Published online: 02 July 2020

\section{References}

1. Li, L. K. et al. Black phosphorus field-effect transistors. Nat. Nanotechnol. 9, 372-377 (2014)

2. Liu, H. et al. Phosphorene: an unexplored $2 \mathrm{D}$ semiconductor with a high hole mobility. ACS Nano 8, 4033-4041 (2014).

3. Castellanos-Gomez, A. et al. Isolation and characterization of few-layer black phosphorus. 2D Mater. 1, 025001 (2014).

4. Koenig, S. P. et al. Electric field effect in ultrathin black phosphorus. Appl. Phys. Lett. 104, 103106 (2014). 
5. Xia, F. N., Wang, H. \& Jia, Y. C. Rediscovering black phosphorus as an anisotropic layered material for optoelectronics and electronics. Nat. Commun. $\mathbf{5}$ 4458 (2014).

6. Tran, V. et al. Layer-controlled band gap and anisotropic excitons in few-layer black phosphorus. Phys. Rev. B 89, 235319 (2014).

7. Yuan, H. T. et al. Polarization-sensitive broadband photodetector using a black phosphorus vertical p-n junction. Nat. Nanotechnol. 10, 707-713 (2015).

8. Chen, $C$. et al. Bright mid-infrared photoluminescence from thin-film black phosphorus. Nano Lett. 19, 1488-1493 (2019).

9. Li, L. K. et al. Direct observation of the layer-dependent electronic structure in phosphorene. Nat. Nanotechnol. 12, 21-25 (2017).

10. Zhang, G. W. et al. Infrared fingerprints of few-layer black phosphorus. Nat. Commun. 8, 14071 (2017).

11. Chen, X. L. et al. High-quality sandwiched black phosphorus heterostructure and its quantum oscillations. Nat. Commun. 6, 7315 (2015).

12. Li, L. K. et al. Quantum oscillations in a two-dimensional electron gas in black phosphorus thin films. Nat. Nanotechnol. 10, 608-613 (2015).

13. Long, G. et al. Achieving ultrahigh carrier mobility in two-dimensional hole gas of black phosphorus. Nano Lett. 16, 7768-7773 (2016).

14. Yang, J. W. et al. Integer and fractional quantum hall effect in ultrahigh quality few-layer black phosphorus transistors. Nano Lett. 18, 229-234 (2018).

15. Ling, X. et al. The renaissance of black phosphorus. Proc. Natl Acad. Sci. USA 112, 4523-4530 (2015).

16. Liu, H. et al. Semiconducting black phosphorus: synthesis, transport properties and electronic applications. Chem. Soc. Rev. 44, 2732-2743 (2015).

17. Chen, X. L. et al. Widely tunable black phosphorus mid-infrared photodetector. Nat. Commun. 8, 1672 (2017).

18. Liu, Y. P. et al. Gate-tunable giant stark effect in few-layer black phosphorus. Nano Lett. 17, 1970-1977 (2017).

19. Yan, S. L. et al. Electrically tunable energy bandgap in dual-gated ultra-thin black phosphorus field effect transistors. Chin. Phys. Lett. 34, 047304 (2017).

20. Deng, B. C. et al. Efficient electrical control of thin-film black phosphorus bandgap. Nat. Commun. 8, 14474 (2017).

21. Chen, $X$. L. et al. Electrically tunable physical properties of two-dimensional materials. Nano Today 27, 99-119 (2019).

22. Wang, Q. H. et al. Electronics and optoelectronics of two-dimensional transition metal dichalcogenides. Nat. Nanotechnol. 7, 699-712 (2012).

23. Huang, L. et al. Waveguide-integrated black phosphorus photodetector for mid-infrared applications. ACS Nano 13, 913-921 (2019).

24. Peng, R. M. et al. Midinfrared electro-optic modulation in few-layer black phosphorus. Nano Lett. 17, 6315-6320 (2017).

25. Whitney, W. S. et al. Field effect optoelectronic modulation of quantumconfined carriers in black phosphorus. Nano Lett. 17, 78-84 (2017).

26. Zhang, R. et al. Broadband black phosphorus optical modulator in the spectra range from visible to mid-infrared. Adv. Optical Mater. 3, 1787-1792 (2015).

27. Guo, Q. S. et al. Black phosphorus mid-infrared photodetectors with high gain. Nano Lett. 16, 4648-4655 (2016).

28. Pei, J. J. et al. Producing air-stable monolayers of phosphorene and their defect engineering. Nat. Commun. 7, 10450 (2016).
29. Yang, J. et al. Optical tuning of exciton and trion emissions in monolaye phosphorene. Light.: Sci. Appl. 4, e312 (2015).

30. Wang, X. M. et al. Highly anisotropic and robust excitons in monolayer black phosphorus. Nat. Nanotechnol. 10, 517-521 (2015).

31. Zhang, S. et al. Extraordinary photoluminescence and strong temperature/ angle-dependent raman responses in few-layer phosphorene. ACS Nano 8 , 9590-9596 (2014)

32. Cui, X. et al. Multi-terminal transport measurements of $\mathrm{MoS}_{2}$ using a van der Waals heterostructure device platform. Nat. Nanotechnol. 10, 534-540 (2015).

33. Britnell, L. et al. Strong light-matter interactions in heterostructures of atomically thin films. Science 340, 1311-1314 (2013).

34. Lee, C. H. et al. Atomically thin p-n junctions with van der Waals heterointerfaces. Nat. Nanotechnol. 9, 676-681 (2014).

35. Withers, F. et al. Light-emitting diodes by band-structure engineering in van der Waals heterostructures. Nat. Mater. 14, 301-306 (2015).

36. Chen, P. et al. Gate tunable $\mathrm{MoS}_{2}$-black phosphorus heterojunction devices. 2D Mater. 2, 034009 (2015).

37. Deng, Y. X. et al. Black phosphorus-monolayer $\mathrm{MoS}_{2}$ van der Waals heterojunction p-n diode. ACS Nano 8, 8292-8299 (2014).

38. Huang, M. Q. et al. Multifunctional high-performance van der Waals heterostructures. Nat. Nanotechnol. 12, 1148-1154 (2017).

39. Liu, X. C. et al. Modulation of quantum tunneling via a vertical twodimensional black phosphorus and molybdenum disulfide $p-n$ junction. ACS Nano 11, 9143-9150 (2017).

40. Liu, Y. P. et al. Tailoring sample-wide pseudo-magnetic fields on a graphene-black phosphorus heterostructure. Nat. Nanotechnol. 13, 828-834 (2018).

41. Qiao, J. S. et al. High-mobility transport anisotropy and linear dichroism in fewlayer black phosphorus. Nat. Commun. 5, 4475 (2014).

42. Sun, Y. et al. Band structure engineering of interfacial semiconductors based on atomically thin lead iodide crystals. Adv. Mater. 31, 1806562 (2019).

43. Cui, Q. N. et al. Transient absorption microscopy of monolayer and bulk WSe ACS Nano 8, 2970-2976 (2014).

44. Castellanos-Gomez, A. et al. Deterministic transfer of two-dimensional materials by all-dry viscoelastic stamping. 2D Mater. 1, 011002 (2014).

45. Yuan, J. T. et al. Photoluminescence quenching and charge transfer in artificia heterostacks of monolayer transition metal dichalcogenides and few-layer black phosphorus. ACS Nano 9, 555-563 (2015).

46. Zhao, W. J. et al. Evolution of electronic structure in atomically thin sheets of $W_{2}$ and $W_{S}$. ACS Nano 7, 791-797 (2013).

47. Yan, R. S. et al. Esaki diodes in van der Waals heterojunctions with broken-gap energy band alignment. Nano Lett. 15, 5791-5798 (2015).

48. Qiu, H. et al. Hopping transport through defect-induced localized states in molybdenum disulphide. Nat. Commun. 4, 2642 (2013).

49. Shim, J. et al. Phosphorene/rhenium disulfide heterojunction-based negative differential resistance device for multi-valued logic. Nat. Commun. 7, 13413 (2016).

50. Favron, A. et al. Photooxidation and quantum confinement effects in exfoliated black phosphorus. Nat. Mater. 14, 826-832 (2015). 